\title{
Scientists slam rationale behind largest HIV vaccine trial
}

The evidence for a combination HIV vaccine is too weak to justify conducting a Phase III trial, says a group of US HIV researchers.

Simon Frantz

A group of prominent US HIV researchers have publicly criticized the scientific rationale behind the largest clinical trial carried out on HIV vaccines.

Twenty-two researchers have written to Science, stating that the US government is wasting its resources in funding a Phase III trial on a combination HIV vaccine, as there is no evidence that either vaccine component works very well on its own (Burton, D. R. et al. Science 303, 316 (2004)).

The trial, called RV144, is a collaboration between the US National Institutes of Health and the Thai government. It has recruited 1,000 subjects in Thailand and aims to recruit 16,000 in total.

RV144 is examining whether a combination of two HIV vaccines will protect against HIV infection and/or control HIV replication through the one-two punch of a prime-boost regimen. But the critics argue that the first 'priming' vaccine, vCP1521 (ALVAC-HIV; Aventis Pasteur), a canarypox virus vector that expresses HIV env, gag and progenes, has shown poor immunogenicity in Phase I and II trials. More culpable, they write, is the use of the second 'booster' vaccine subunits of the HIV surface glycoprotein gp120 (AIDSVAX B/E; VaxGen) - as this lacked efficacy in Phase III trials.

Combining the two doesn't add up, says Michael Lederman, professor of medicine at the Case Western Reserve University School of Medicine, and co-author of the article.

“The vaccine's immunogens aren't very good, and we are concerned that a trial of this size is being carried out which won't have a reasonable chance of success," he says.

The US National Institute of Allergy and Infectious Diseases (NIAID), which inherited the RV144 trial from the US Department of Defense, says that there are important reasons for carrying out the trial. "We still have no idea of what immune responses provide protection against HIV or reduce viral load," says Margaret Johnston, director of the Vaccine and Prevention Research Program for NIAID's Division of AIDS. "So, there is a very high probability that information gained from this trial will advance HIV vaccine development."

Development of an HIV vaccine is hampered by the fact that no one is known to have recovered from, and cleared, acute HIV

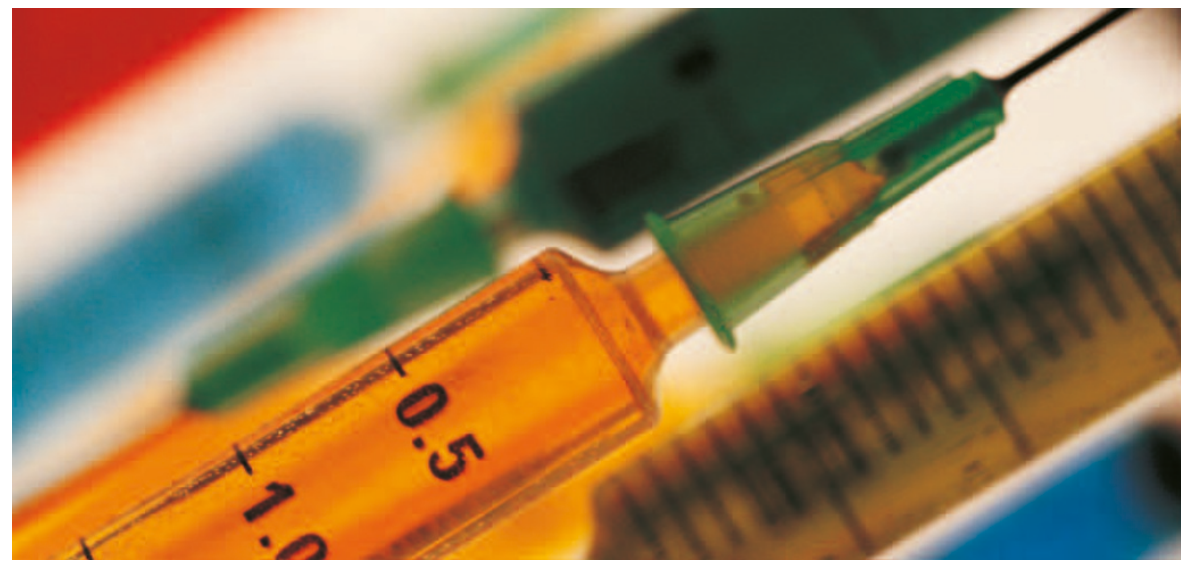

A Phase III trial on a combination vaccine for HIV is at the centre of a hot dispute.

infection. However, what is known is that both arms of the immune system make a valiant attempt to control the virus. The humoral response produces antibodies that neutralize HIV activity, and the cell-mediated response produces cytotoxic T lymphocytes (CTLs), which destroy HIV-infected cells and halt HIV replication.

vCP1521 targets the cell-mediated immune response and gp120 targets the humoral response, and if combined could therefore form a double-pronged attack on the virus. "As you are trying to combat HIV diversity, a greater breadth of response may allow for a better effect," says Johnston.

\section{"... there is no evidence that either vaccine component works very well on its own."}

The critics contend that the objectives of the trial are unlikely to be achieved. In February 2003, more than a year after RV144 was approved, results from Phase III trials of gp120 in 5,000 subjects showed no difference in infection rates compared with placebo, apart from in a much-disputed positive subgroup analysis of 13 black and 4 Asian subjects who became infected with HIV. "There is no credible scientific justification for the inclusion of gp120 in the trial," says John Moore, professor of microbiology and immunology at Cornell University, New York, and co-author of the article. "It's an expensive, inert component that complicates any analysis of the final outcome."

In their reply to the Science letter, Johnston and colleagues from NIAID and the Walter Reed Army Institute of Research,
Washington, DC, argue that the trial should still be conducted because the efficacy of gp120 alone cannot predict its contribution to the vCP1521/gp120 vaccine (McNeil, J. G. et al. Science 303, 961 (2004)). They write that although arguably modest, early studies have shown that the combination augments immune responses relative to each vaccine alone. The combination vaccine induced CTL responses in $25-45 \%$ of recipients, and there were qualitative changes in $\mathrm{CD}^{+}$ $\mathrm{T}$-cell response, which stimulates several immune responses, such as generation of neutralizing antibodies. Antibody-dependent cellular cytotoxicity was also induced.

But the critics say that much of this evidence is too weak to justify a Phase III trial. An enhanced response could be measured rapidly by a smaller trial. How much of a response is enough to justify a trial is a reasonable question on which reasonable people will have to disagree on, says Johnston. "No one really knows how high the bar needs to be, so why not let experiments provide the data to answer these questions. Some say 'more than the last one' is sufficient to move ahead, citing that vaccine development is incremental."

Johnston says that changing the design of the trial at this point might not be feasible. But, as a result of the letter Johnston and colleagues will broaden discussion on the investigation of the immune response. This comes as good news to Moore. "It was to try to improve the trial design and the quality of the information that might be learned that the letter was written. There are now indications that our concerns have been recognized, and that there will be some improvements. Let's hope that that is what actually happens." 